EPJ Web of Conferences 103, 03010 (2015)

DOI: 10.1051/epjconf/201510303010

(C) Owned by the authors, published by EDP Sciences, 2015

\title{
Quantum Memory as Light Pulses Quantum States Transformer
}

\author{
A.N.Vetlugin ${ }^{a}$ and I.V. Sokolov \\ Saint Petersburg State University, 7-9 Universitetskaya nab., St. Petersburg 199034 Russia
}

\begin{abstract}
Quantum memory can operate not only as a write-in/readout device [1] for quantum light pulses and non-classical states generation [2] device but also as a quantum states of light transformer. Here the addressable parallel quantum memory [3] possibilities for this type of transformation are researched. Quantum memory operates as a conventional N-port interferometer with $\mathrm{N}$ equals to the number of the involved spin waves. As example we consider the ability to transform quantum states of two light pulses - in this case the quantum memory works as a mirror with a controlled transmission factor.
\end{abstract}

Keywords: quantum optics, quantum information, quantum memory, squeezed and entangled states, spin waves.

In the last 5-10 years the great progress in experimental realization of quantum memory protocols was achieved. The high quantum memory parameters - the efficiency up to $87 \%$ [4], the fidelity up to 0.999 [5], the storage time up to one minute [6], the simultaneous storage of 1060 temporal modes allows one to talk about the quantum memory implementation un quantum information protocols such as quantum repeaters, quantum computation, etc. [8]. Besides the quantum states of light storage the quantum memory can be used for non-classical states of light generation (entangled and squeezed) for using in discussed protocols. Here we research an ability to use the quantum memory as a transformer for input quantum light pulses. The transformation is achieved by write-in and readout of a set of modes (temporal, frequency or spatial) of light and their mixture due to simultaneous treatment to different modes.

The quantum memory scheme (Figure 1) is an ensemble of cold atoms with spin-1/2 both in ground and excited states in a constant magnetic field. The ensemble is placed in a ring cavity that supports one longitudinal mode. The quantum light pulse comes to the input mirror. Conditions for efficient write-in/readout processes in time-reversal and impedance matching approaches are discussed in $[3,9]$. We assume that the high-efficiency regimes are used, what is achieved by long enough (compared to cavity field lifetime) time duration of signals. The quantum memory addressability researched in [3] allows us to write-in the quantum light pulses succession to one quantum memory cell and retrieve these pulses in required order. This is achieved by the fact that for particular pump wave direction the only one spin wave is involved to the interaction with light.

\footnotetext{
${ }^{a}$ Corresponding author: vetluginanton@gmail.com
} 


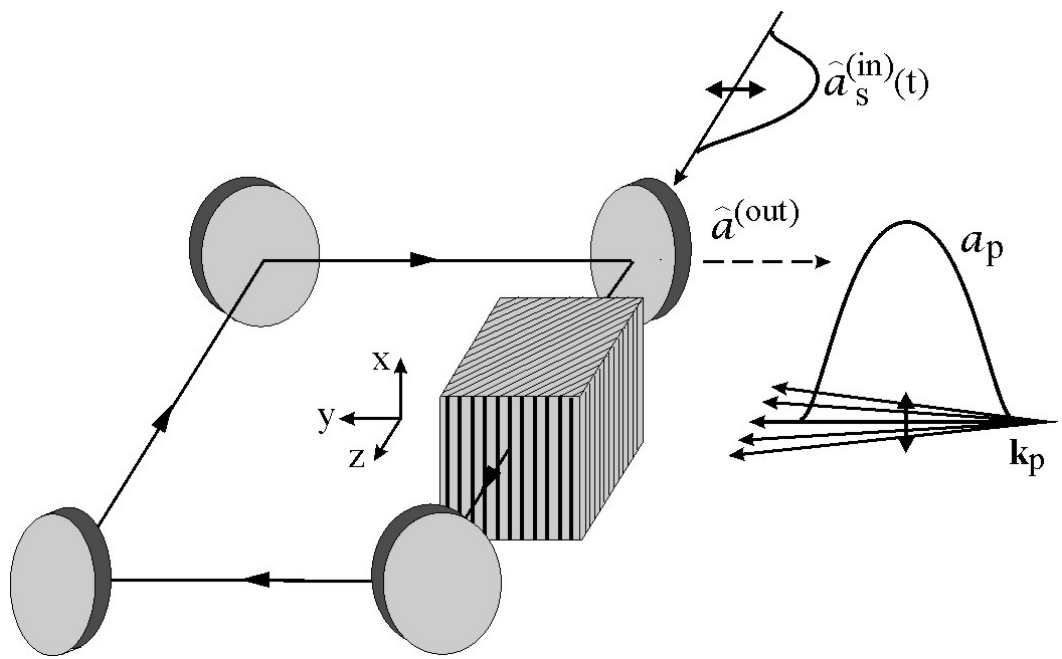

Figure 1.The parallel cavity-based quantum memory scheme: ring cavity with atomic ensemble (the cube on the figure) inside, the quantum light pulse comes to the input port, pump wave propagates in $\{\mathrm{y}, \mathrm{z}\}$ plane.

Here we develop ideas proposed in [3]. We consider the case of many pump waves (each pump waves is responsible for one independent spin wave evolution) are directed to quantum memory cell on the readout stage. The cavity field is excited by many spin waves at once - as a result the output light pulse consists of input signals mixture. The "mixture factors" are determined by the pump waves amplitudes. If we know the input light pulses states and adjust the pump waves amplitudes we can control the output light pulses states.

As an example we consider the quantum memory operation as a mirror with controlled transmission factor for two input light pulses. We show the ability to create entangled states of two light pulses from two squeezed in the orthogonal quadratures input light pulses, and vice versa.

The ability to manipulate the light pulses states parallel with efficient write-in, readout and nonclassical states generation inside the quantum memory allows one to simplify quantum information protocols and raise their efficiency.

\section{References}

1. A.I. Lvovsky, B.S. Sanders, W. Tittel. Nat. Photonics 3, 706 (2009)

2. L.-M. Duan, M.D. Lukin, J.I. Cirac and P. Zoller. Nature 414, 413-418 (2001)

3. A.N. Vetlugin and I.V. Sokolov. Eur. Phys. J. D 68, 269 (2014)

4. M. Hosseini, B. M. Sparkes, G. Campbell, P. K. Lam and B. C. Buchler. Nat. Commun. 2, 174 (2011)

5. Z.-Q. Zhou, W.-B. Lin, M. Yang, C.-F. Li, and G.-C. Guo. Phys. Rev. Lett. 108, 190505 (2012)

6. G. Heinze, C. Hubrich, and T. Halfman. Phys. Rev. Lett. 111, 033601 (2013)

7. M. Bonarota, J.-L. Le Gouet, and T. Chaneliere. New J. Phys. 13, 013013 (2011)

8. F. Bussieres, N. Sangouard, M. Afzelius, H. de Riedmatten, C. Simon and W. Tittel. J. Mod. Optics 60 (18), 1519 (2013)

9. A.N. Vetlugin and I.V. Sokolov. Opt. Spectr. 115 (6), 980 (2013) 